\title{
Manipulation of Droplets onto a Planar Interface
}

T. Gilet, D. Terwagne, N. Vandewalle and S. Dorbolo

GRASP, Département de Physique B5, Université de Liège, B-4000 Liège, Belgium

Contents

A. Introduction . . . . . . . . . . . . . . . . . . . . . . . . . 503

B. Delayed Coalescence on a Planar Interface at Rest . . . . . . . . . . . . . . . . . . . . . 504

1. Reynolds Model . . . . . . . . . . . . . . . . . . . . . . . . . . . 505

2. Other Influences . . . . . . . . . . . . . . . . . . . . . . . . . . . . . . . . . . . . . . . . . . . . . . . . . . .

C. Delayed Coalescence on a Vertically Vibrated Bath . . . . . . . . . . . . . . . . . . . 506

1. Experimental Setup . . . . . . . . . . . . . . . . . . . . . . . . . . 507

2. Lifetime of Droplets . . . . . . . . . . . . . . . . . . . . . . . . . . . . . 508

3. Trajectory and Deformation of Droplets . . . . . . . . . . . . . . . . . . . 509

D. Partial Coalescence . . . . . . . . . . . . . . . . . . . . . . . . . . . . 510

1. Dimensional Analysis . . . . . . . . . . . . . . . . . . . . . . . . 511

2. The Self-Similar Regime . . . . . . . . . . . . . . . . . . . . . . . . . 512

3. Influence of Gravity . . . . . . . . . . . . . . . . . . . . . . . . . . . . 513

E. Influence of Viscosities . . . . . . . . . . . . . . . . . . . . . . . . . . 514

F. Mixing Droplets Together . . . . . . . . . . . . . . . . . . . . . . . . 516

G. Control of the Partial Coalescence with Bouncing . . . . . . . . . . . . . . . . . . 517

H. Conclusion . . . . . . . . . . . . . . . . . . . . . . . . . . . . 518

I. Acknowledgements . . . . . . . . . . . . . . . . . . . . . . . . . . . . . 519

J. References . . . . . . . . . . . . . . . . . . . . . . . . . . . . . . . . . 519

\section{A. Introduction}

The manipulation of droplets becomes a huge problem as soon as tiny quantities of liquid have to interact with micro-sensors or with another liquid. A broad range of applications can be found in food science, pharmacology, coating, painting and biotechnology among others. Droplets can be used in chemical engineering in order to mix tiny amounts of reactive substances [39]. To achieve such a goal, one has to invent some processes to manipulate a droplet: motion, binary collision, fragmentation, ... Most of efforts were made in the framework of microfluidic [1], i.e. the droplets are driven through close channels using a second liquid (nonmiscible with the liquid of the droplet). That technique requires a perfect control of the surface properties: wetting, de-wetting, pinning of the contact line, ... Accurate 
pumps are needed to drive the droplet through the network of channels. Finally, the 1 contamination should be prevented by avoiding any contact with a solid element. 2 An alternative technique to the microfluidic channels consists in manipulating indi- 3 vidual droplets onto a liquid bath.

Couder et al. have shown that a droplet is able to bounce indefinitely on the surface of a liquid pool without coalescing [2]. This effect is basically obtained when the liquid bath is vertically shaken. Moreover, he demonstrated that the droplet moves horizontally due to its interaction with the wave it produces on the liquid surface [3]. This wave-particle interaction reveals some spectacular behaviours including interference patterns [4] and tunnelling effects [5].

In the present work, we describe several operations that can be performed with droplets onto a planar interface, including rebound, division, mixing and size selection. All these operations are done without touching the droplets. By vibrating the bath, a droplet can be stored. The stability of the bouncing droplet is discussed in Section C. Using the phenomenon of partial coalescence, it is possible to decrease the volume of a droplet (Section D). We discuss the best way to mix two droplets (Section E). Finally, by combining the delayed coalescence and the partial coalescence, we show how to have a control on the droplet size (Section F). In the next section, the mechanism related to the delay before coalescence of a droplet with a planar interface is described. This section establishes the theoretical basis of this work.

\section{B. Delayed Coalescence on a Planar Interface at Rest}

Let us consider a system composed of two immiscible fluids (at least one of them is a liquid): fluid 1 is heavier than fluid 2 . A droplet made of fluid 1 is gently laid onto the quasi-planar interface between both fluids. The droplet tends to cross this interface due to gravity: it experiences a coalescence. 3

Figure 1. Sketch of a droplet of radius $R$ made of a liquid 1. This droplet is surrounded by a fluid 2 that is not miscible with fluid 1 (may be air). The droplet is laid onto the planar interface between fluid 1 and fluid 2. The coalescence occurs when the interstitial film of fluid 2 (thickness $h$ ) breaks.

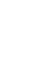

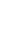

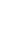

8

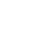

(1)

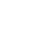

4

6
19

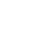

4

26

7

8

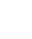


The merging does not occur immediately. Indeed, the film of fluid 2 that remains between the droplet and its bulk phase has to be drained out. Then, when this film is sufficiently thin, it is broken by attractive forces (Van Der Waals interactions, hydrogen bonds, ...). The drainage time of the film is influenced by a lot of physical parameters that are discussed here below.

\section{Reynolds Model}

The first model that describes the drainage before coalescence was proposed by Reynolds [6]. It is based on the lubrication theory: the film of fluid 2 is sufficiently thin compared to the droplet radius $R$, inertia effects are neglected, and both fluids are Newtonian and incompressible. Reynolds supposes that the film thickness $h$ is constant, and that both interfaces are motionless. This latter hypothesis is approximately satisfied when the dynamical viscosity $\mu_{2}$ of fluid 2 is much smaller than the viscosity of fluid 1 , so the flow inside the film cannot entrain the fluid inside the droplet. In these conditions, the lifetime $t_{\mathrm{L}}$ of the droplet onto the planar interface, due to drainage, is given by:

$$
t_{\mathrm{L}}=\frac{9}{16 \pi^{2}} \frac{\mu_{2} S^{2}}{\Delta \rho g R^{3} h_{\mathrm{c}}^{2}},
$$

where is $\Delta \rho$ the difference in density between both fluids, $g$ the gravity acceleration and $h_{\mathrm{c}}$ the critical film thickness for which electrostatic forces become significant. The area of the film $S$ depends on the geometry of the quasi-equilibrium configuration that is reached when the droplet settles on the planar interface.

Usually, $S / R^{2}$ is a monotonic function of the Bond number $B o$, the ratio between gravity and surface forces (interfacial tension $\sigma$ ):

$$
B o=\frac{\Delta \rho g R^{2}}{\sigma} .
$$

Moreover, when $B o \ll 1$, the droplet is approximately spherical onto a planar interface, $S / R^{2} \sim 2 \pi B o / 3$ and the resulting lifetime is

$$
t_{\mathrm{L}}=\frac{1}{4} \frac{\mu_{2} \Delta \rho g R^{5}}{h_{\mathrm{c}}^{2} \sigma^{2}} .
$$

For a millimetric droplet of water in the air laid onto a water surface, a lifetime of about $1 \mathrm{~s}$ is found, taking $h_{\mathrm{c}}=100 \mathrm{~nm} \mathrm{[7,8]}$.

\section{Other Influences}

The observed lifetime is often smaller than the predicted one. The model of Reynolds has been improved by several authors. For example, more realistic geometrical configurations are detailed in [9]. Another important point is the interfacial mobility: When dynamical viscosities are similar in both fluids, the fluid inside the droplet is entrained by the flow in the film. This entrainment can be reduced when surfactant is added, since it usually rigidifies the interface. An advanced investigation about the impact of interfacial mobility has been made by Ivanov et al. [10]. 
Other effects, such as Marangoni effects, rheological interfacial properties, ther- 1 mocapillary effects [11], electrocoalescence [12] have been studied in details. The 2 interested reader could refer for example to the review of Neitzel and Dell' Aversana 3 [11].

\section{Delayed Coalescence on a Vertically Vibrated Bath}

Couder et al. [13] have recently discovered that when a droplet is laid on an interface that is vertically vibrated (by using an electromagnetic shaker for example Fig. 2), it may avoid coalescence. Indeed, when the droplet bounces, the air film is renewed. The oscillation is characterised by the reduced acceleration $\Gamma$ defined by

$$
\Gamma=\frac{A \omega^{2}}{g},
$$

where $A$ and $\omega$ are the amplitude and pulsation of the sinusoïdal oscillation; $g$ is the gravity acceleration.

Sustained bouncing is observed when the acceleration is larger than a critical acceleration $\Gamma_{\mathrm{C}}$ given by [13]

$$
\Gamma_{\mathrm{C}}-1 \propto \omega^{2} .
$$

This criterion allows us to determine whether the droplet bounces or not according to a couple of parameters $(A, \omega)$ of the oscillation. Note that this criterion is only valid for high viscosity oil and when the viscosities of both the bath and the droplet are the same. Indeed, we showed that for low viscosity systems, the droplet deformations must be taken into account as eigen modes are excited [14, 15].

Stabilisation by bouncing is limited by the appearance of Faraday waves. This high acceleration limit $\Gamma_{\mathrm{F}}$ depends on the viscosity of the oil in the bath. Considering a given droplet size and a given viscosity, the bouncing phenomenon may only occur in a delimited region of the $(A, \omega)$ diagram, i.e. in between $\Gamma_{\mathrm{C}}$ and $\Gamma_{\mathrm{F}}$ (Fig. 3).

A question remains about the stability of the bouncing droplet. Indeed, at low frequencies and for $\Gamma>\Gamma_{\mathrm{C}}(\omega)$, the droplet may bounce for a time longer than
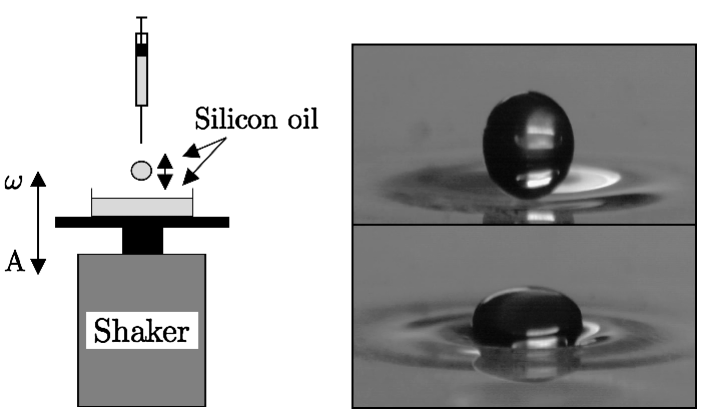

Figure 2. (left) Experimental setup made up of an electromagnetic shaker and a plate on which an oil-filled container is fixed; (right) snapshots of the bouncing droplet. 


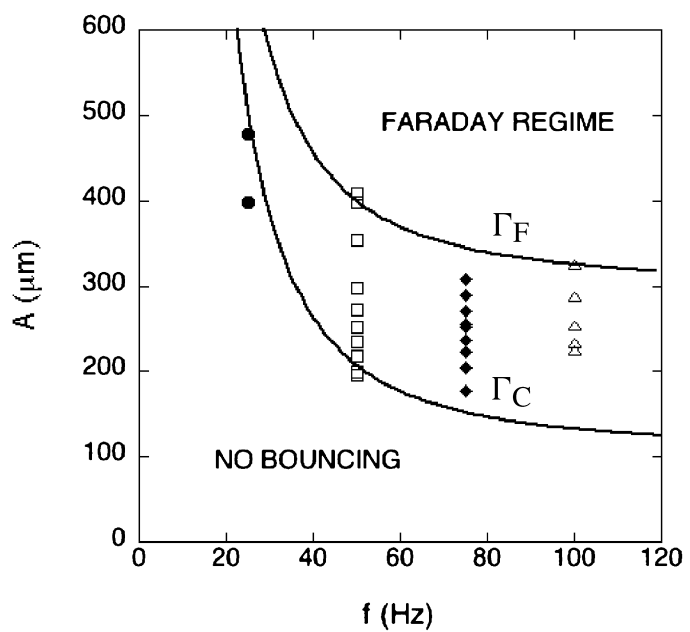

Figure 3. Phase diagram amplitude-frequency for a droplet of $1.5 \mathrm{~mm}$ of diameter and made up of $50 \mathrm{cSt}$ silicone oil. Top and bottom curves correspond to the Faraday $\Gamma_{\mathrm{F}}$ and Couder $\Gamma_{\mathrm{C}}$ acceleration thresholds respectively (see text). Points represent experimental conditions that have been investigated by Terwagne et al. [16] in order to cover at best the region where the droplet can bounce.

a few days. On the other hand, for large frequencies and for $\Gamma>\Gamma_{\mathrm{C}}(\omega)$, the droplet bounces only for a few seconds before coalescing. Let us determine where, in the phase diagram $(A, \omega)$, the droplet bounces the longest time.

Due to the large number of parameters: $A, \omega, R$ and the viscosity of the oil $\mu_{1}$, some parameters have been arbitrary fixed. The viscosity and the droplet size are fixed to $50 \mathrm{cSt}$ and $1.5 \mathrm{~mm}$ diameter respectively. In these conditions, the area to explore in the $A-\omega$ diagram and the investigation points are represented in Fig. 3. For each point, the lifetime has been measured. Moreover, an optical tracking method has been used to determine the position and the deformation of the droplet with respect to the phase of the plate.

\section{Experimental Setup}

A container is filled with Dow Corning silicone oil (DC200) which viscosity $\mu_{1}=50 \mathrm{cSt}$. For each frequency, the acceleration is tuned between $\Gamma_{\mathrm{C}}$ and $\Gamma_{\mathrm{F}}$ by increasing the amplitude of the vibration. The droplets are produced by using a syringe and are laid onto the surface of the bath. The lifetime is basically measured using a timer.

The position and the shape of the droplets have been studied by image processing. The motion is recorded from the side with a high-speed camera (1000 fps). A snapshot of an impacting droplet is shown in Fig. 4. During bouncing, the deformation is vertically asymmetric. The evolution of its upper half part is easily determined by a contour detection on the images while its lower part is not accessible at any time of the motion, in particular when the droplet is partly immersed in the bath (Fig. 4). 


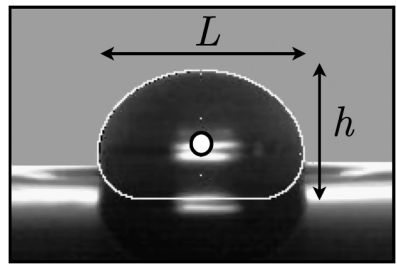

Figure 4. Snapshot of a droplet at its maximum of deformation. The fitted contour of the droplet is represented by a white curve. The height $h$ and the width $L$ are indicated by the arrows while the centre of mass is shown by a white circle.

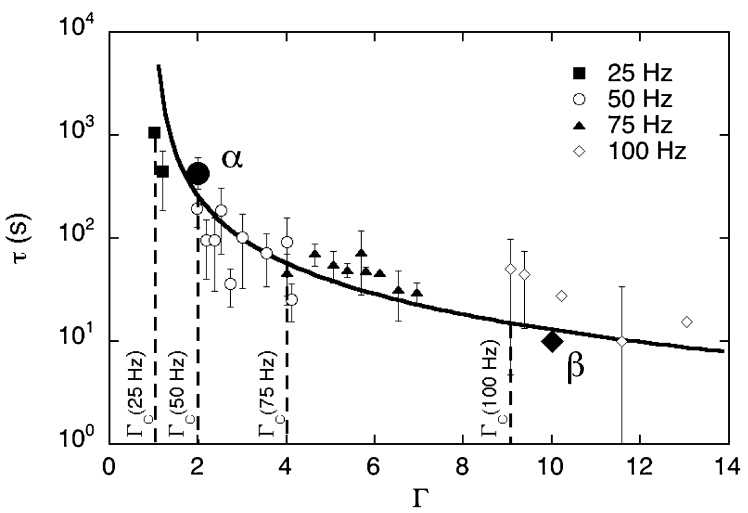

Figure 5. Semi-log plot of the averaged lifetime $\tau$ as a function of the reduced acceleration $\Gamma$. The symbols correspond to the different frequencies (see legend). The vertical dashed lines represent the Couder threshold Eq. (5) corresponding to the different frequencies. The continuous curve is the fit using Eq. (6). The $\alpha$ and $\beta$ symbols refer to the cases that have been studied according to their trajectories.

However, since the total volume and the evolution of the upper part of the droplet are known, the lower part may be deduced as soon as a hypothetical shape has been speculated. The lower part shape is modelled by a flat bottom surrounded by a quarter of torus. The shape is fitted to the experimental contour, taking volume conservation into account. An example of this shape (white contour) can be seen in Fig. 4. On the other hand, when the droplet is entirely visible, its shape is oblate. The lower part of the droplet is supposed to behave like a half ellipsoid. Eventually, the trajectory of the centre of mass is deduced as well as the evolution of the aspect ratio $A_{\mathrm{r}}=$ $h / L$, where $h$ and $L$ are respectively the height and the width of the droplet (Fig. 6).

When the amplitude of the oscillation is not sufficiently large, the air film below the droplet is not completely regenerated and the droplet coalesces instantaneously.

\section{Lifetime of Droplets}

Even beyond $\Gamma_{\mathrm{C}}$, we observe that the lifetime of bouncing droplets is finite. This later gives us some information about the stability of bouncing. The lifetime is represented in Fig. 5 with respect to the reduced acceleration $\Gamma$. Different symbols 
correspond to the considered frequency, i.e. circles, squares, diamonds and triangles are for 25, 50, 75 and $100 \mathrm{~Hz}$ respectively. The vertical dashed lines represent the Couder threshold $\Gamma_{\mathrm{C}}(\omega)$.

The lifetime is found to decrease with an increase in $\Gamma$. The lifetime has been represented in a semi-log plot. A power law has been fitted (continuous curve in Fig. 5), namely

$$
\tau \propto \frac{1}{(\Gamma-1)^{1.4}} .
$$

A divergence is expected at $\Gamma=1$. Lifetimes at $25 \mathrm{~Hz}$ are difficult to determine since droplets last for a very long time.

As far as the results corresponding to a given frequency are concerned, the maximum lifetime is found at $\Gamma_{\mathrm{C}}$. When the acceleration is increased above the threshold, the droplet deformations increase and destabilize the bouncing. It results in a shorter lifetime.

In the next section, the deformation is finely studied for two different cases: (i) case $\alpha$ : a bouncing droplet characterized by a lifetime of $420 \mathrm{~s}(A=200 \mu \mathrm{m}$ and $f=50 \mathrm{~Hz}$ ) and (ii) case $\beta$ : a bouncing droplet characterized by a lifetime of $10 \mathrm{~s}$ $(A=250 \mu \mathrm{m}$ and $f=100 \mathrm{~Hz}$ ).

\section{Trajectory and Deformation of Droplets}

In Fig. 6, the cases $\alpha(\bullet)$ and $\beta(\diamond)$ are compared: The vertical position of the centre of mass, normalized by $A$, is plotted on Fig. 6(a) while Fig. 6(b) presents the variations of the aspect ratio with respect to the normalized time $t_{\mathrm{r}}=\omega t / 2 \pi$. In both
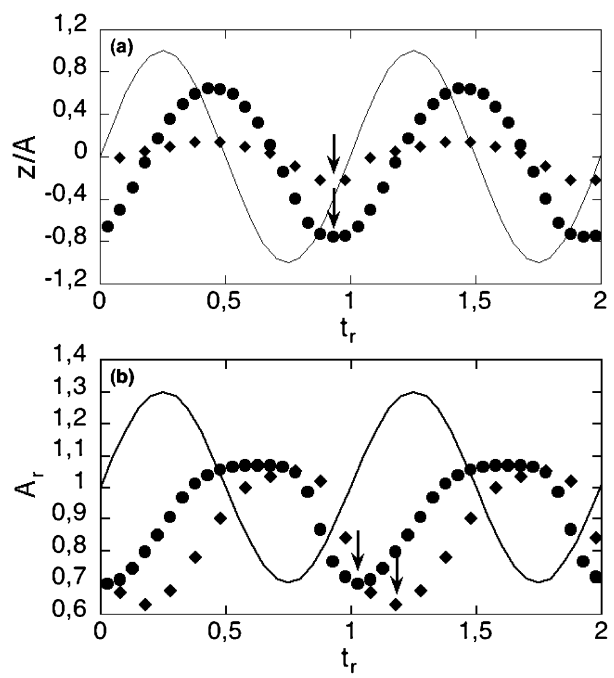

Figure 6. Comparison of (a) the position of the centre of mass and (b) the aspect ratio variation with respect to the reduced time $t_{\mathrm{r}}=\omega t / 2 \pi$ for the case $\alpha$ and $\beta$ (see text). These cases are represented by circles and squares respectively. The continuous curve gives an indication concerning the phase of the plate. 
figures, continuous curves represent the position of the plate in arbitrary units in order to visualize the phase of the plate.

In both cases, the motion seems to be perfectly periodic. The normalized position of the droplet is characterized by a larger amplitude in the low frequency case. However, the phase $t_{\mathrm{r}, \mathrm{z}}$ when the trajectory reaches a minimum is nearly the same in both cases. This particular phase is indicated by an arrow in Fig. 6(a). The trajectory in the case $\alpha$ is more symmetrical than in the case $\beta$.

The variation of the aspect ratio has nearly the same amplitude in both cases, but the phase $t_{\mathrm{r}, \mathrm{Ar}}$ of maximum deformation is observed to be very different. This particular phase is indicated by an arrow in Fig. 6(b). The bouncing droplet is deformed during a smaller laps of time in case $\alpha$ than in the case $\beta$. Indeed, the aspect ratio $A_{\mathrm{r}}$ in case $\alpha$ is close to and even higher than unity during a longer reduced time. That means that the time of interaction between the bath and the droplet is smaller than in case $\beta$. In that later case, the droplet is continuously deformed by the plate.

In view of these results, a long lifetime is characterized by a large amplitude motion of the centre of mass and a long period without interaction with the bath. Moreover, the phase at which the maximum deformation occurs is of importance. The ideal situation is obtained when $t_{\mathrm{r}, \mathrm{z}} \sim t_{\mathrm{r}, \mathrm{Ar}}$.

\section{Partial Coalescence}

A droplet made of a low-viscosity liquid can experience a partial coalescence when it is gently laid onto a liquid bath [1]: it does not fully empty and a new smaller droplet is formed above the bath interface (Fig. 7). This droplet coalesces partially again and the process repeats until a critical size is reached. Then, the coalescence becomes total and the last droplet fully disappears.

This phenomenon was first reported in 1930 by Mahajan [18], and investigated by Charles and Mason in 1960 [19]. In their experiments, another immiscible liquid was surrounding the droplet (instead of air). No more investigations were made before those of Leblanc [20] in 1993. He was interested in the stability of emulsions. Indeed, partial coalescence considerably slows down the gravity-driven phase separation of two immiscible liquids, for instance when dealing with oil demulsification. Unfortunately, his work remained unpublished. In 2000, Thoroddsen and Takehara [21] studied this phenomenon in more details for an air/water interface. In the same time, partial coalescence with a surfactant addition was described by Pikhitsa and Tsargorodskaya [22]. In 2006, many investigations were conducted in order to fully understand partial coalescence (Blanchette et al. [23], Kavehpour et al. [24, 25], Feng et al. [26, 27]). Finally, an experimental study of the influence of both viscosities on the process has been made by Gilet et al. [28].

Until now, little is known about micro-flows occurring during coalescence. First, traditional experimental techniques are difficult to be applied at droplet scale. PIV experiments on droplet coalescence were made [29]. Unfortunately in [29], the 

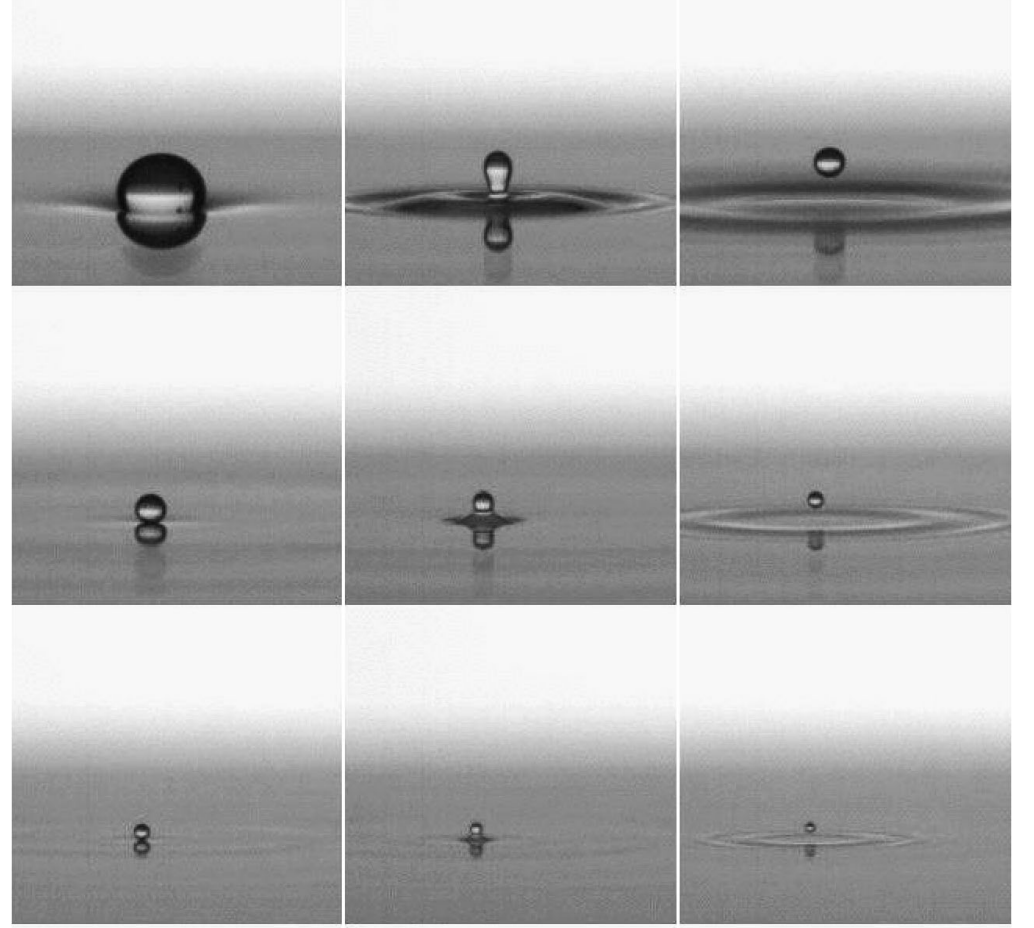

Figure 7. A soapy water droplet is gently laid onto a soapy water bath. The droplet partially coalesces, and a new smaller droplet is formed. This phenomenon occurs several times. When the droplet is sufficiently small, the coalescence becomes total. The arrow on the last snapshot indicates the position of the last micro-droplet before its total coalescence.

droplet was too large to allow partial coalescence. The simplest way to get experimental information about micro-flows is to observe the interface position. From a numerical point of view, the free surface flows with changing topology (such as coalescence, breakdown and pinch-off) are really difficult and expensive to compute. Despite these difficulties, a lot of information has already been collected about micro-flows in coalescence [23, 27].

\section{Dimensional Analysis}

Charles and Mason [19] attempted to explain the occurrence of partial coalescence by considering the ratio of dynamical viscosities in both fluids. But this only pa- 
rameter is not sufficient to fully understand partial coalescence mechanisms. As 1 discovered by Leblanc [20], three kinds of forces influences the process dynamics: 2 the reduced gravity, the surface tension and the viscosity in both fluids. 3

Partial coalescence is usually scaled by the capillary time $\tau_{\sigma}[21,28,30]$, result- 4 ing from the balance between interfacial tension and inertia:

$$
\tau_{\sigma}=\sqrt{\frac{\rho_{\mathrm{m}} R^{3}}{\sigma}},
$$

where $\rho_{\mathrm{m}}$ is defined as the mean density $\left(\rho_{1}+\rho_{2}\right) / 2$ and $R$ is the radius of the initial droplet. Therefore, the interfacial tension has to be the main force for partial coalescence to occur. In other words, a self-similar process such as partial coalescence is only possible when a single force is dominant (here the surface tension), there is thus no natural length scale related to the balance of two forces.

Reduced gravity is compared to surface tension by means of the Bond number Bo, as mentioned previously. To assess about the influence of viscosities, one can use the Ohnesorge numbers, i.e. the ratios between $\tau_{\sigma}$ and the viscous times $R^{2} / v$ in both fluids

$$
O h_{1,2}=\frac{\nu_{1,2} \sqrt{\rho_{\mathrm{m}}}}{\sqrt{\sigma R}},
$$

where $v_{1,2}$ is the kinematic viscosity of the fluid 1 and 2 respectively. According to the $\pi$-theorem (Vaschy-Buckingham), a fourth dimensionless number is required in order to describe the whole system. It could be the relative difference of density,

$$
\Delta \rho=\frac{\rho_{1}-\rho_{2}}{\rho_{1}+\rho_{2}}
$$

linked to the relative difference of inertial effects generated by the interfacial tension in both fluids. Ideally, any dimensionless quantity can be expressed as a function of these dimensionless numbers, especially the ratio $\Psi$ between the daughter droplet radius $R_{\mathrm{d}}$ and the mother droplet radius $R$

$$
\frac{R_{\mathrm{d}}}{R}=\psi\left(B o, O h_{1}, O h_{2}, \Delta \rho\right) .
$$

\section{The Self-Similar Regime}

When $B o \ll 1$ and $O h_{1,2} \ll 0.1$, gravity and viscosities have no significant influence on the process $[21,23,28]$. The $\Psi$ function does not depend on $B o$ and $O h_{1,2}$ anymore: it becomes a function $\Psi_{0}$ that only depends on $\Delta \rho$. In these conditions, the process is self-similar since the densities do not change between two successive partial coalescences. In liquid/liquid systems, the difference of density is small and $\Psi_{0}$ is approximately equal to $0.45[21,23,28]$.

In Fig. 8, the successive stages of the partial coalescence are captured with a high-speed camera. The shape evolution of the interface is relatively constant from one experiment to another [28]. 


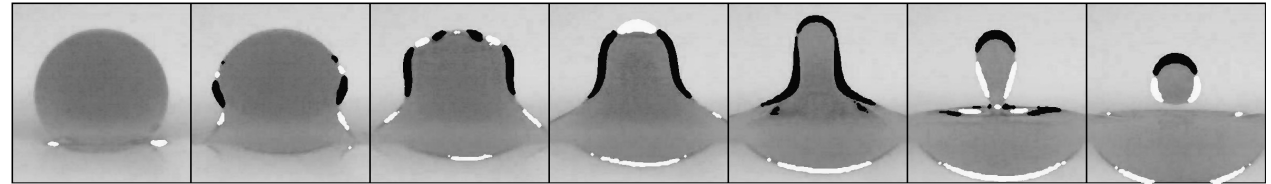

Figure 8. Different stages of a partial coalescence in the self-similar regime. Capillary waves are highlighted by black regions (receding interface) and white regions (progressing interface).

The film rupture occurs where the film is the thinnest. Since the surrounding fluid generates an overpressure (and thus a dimple) at the centre while it is drained outward, the thinnest point is off-centre $[9,31]$. When the film is broken, it quickly opens due to high pressure gradients created by surface tension near the opening [32-35].

The emptying droplet takes a column shape, and then experiences a pinch-off that leads to the formation of the daughter droplet. Charles and Mason [19] have suggested that this pinch-off was due to a Rayleigh-Plateau instability. Recently, Blanchette and Bigioni [23] have shown that this hypothesis was wrong. They have solved Navier-Stokes equations for a coalescing water droplet surrounded by air. When the top of the droplet reaches its maximum height, they stop the numerical simulation, set velocities and pressure perturbations to zero, and restart the computation. The coalescence, normally partial, was observed to be total with this flow reset. The pinch-off cannot be due to a Rayleigh-Plateau instability.

During a coalescence, capillary waves are generated at the bottom of the droplet after the film breakdown [20, 21, 29]. A part of them propagates far away, on the planar interface. The other part climbs over the droplet and converges at the top. Those waves are visible in Fig. 8, where the progressing/receding feature of the interface is highlighted. According to Blanchette [23], such a convergence at the top greatly deforms the droplet and delays its coalescence: the horizontal collapse (the pinch-off) can occur before the emptying. Therefore, the pinch-off that enables partial coalescence seems to be mainly due to the capillary waves. The pinch-off usually occurs between $1.4 \tau_{\sigma}$ and $2 \tau_{\sigma}$ after the film breakage. After that, the fluid creates a vortex ring that goes down through the fluid 1 [36-38].

\section{Influence of Gravity}

For large droplets, gravity is known to be as important as surface tension $(B o \sim 1)$. Therefore, gravity significantly accelerates the emptying of the mother droplet $[20,23]$. Moreover, it flattens the initial droplet. When viscosities are negligible $\left(O h_{1,2}<7.5 \cdot 10^{-3}\right)$, the ratio $\Psi$ is monotonically decreasing with $B o$ (Fig. 9), and it suggests that a critical Bond number $B o_{\mathrm{c}}$ should exist for which $\Psi=0$ for $B o>B o_{\mathrm{c}}$ : coalescence becomes total. Mohamed-Kassim and Longmire [29] have reported total coalescences for Bond numbers equal to 10. However, both Ohnesorge numbers were greater than 0.01 , and viscosity played a significant part on the outcome of these coalescences. Until now, no experiment assesses about the existence of $B o_{\mathrm{c}}$ for negligible viscosities. 


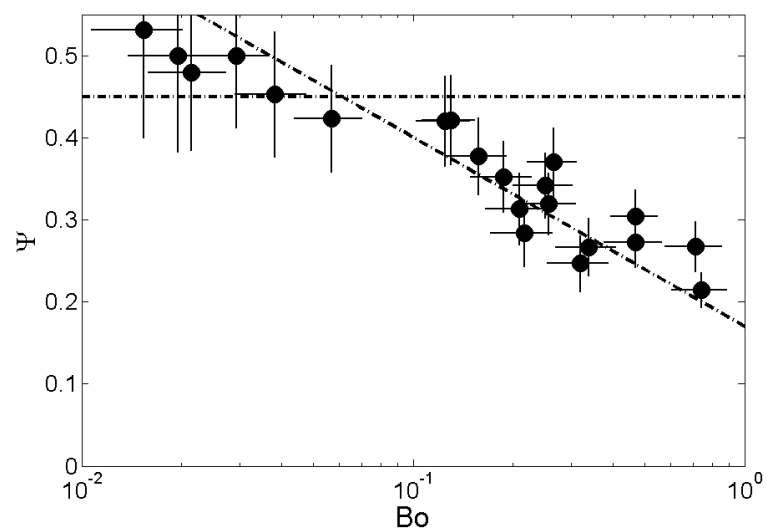

Figure 9. Semi-log plot of the radius ratio $\Psi$ as a function of the Bond number Bo when 13 $O h_{1,2}<0.0075$. Dashed lines are guides for the eyes.

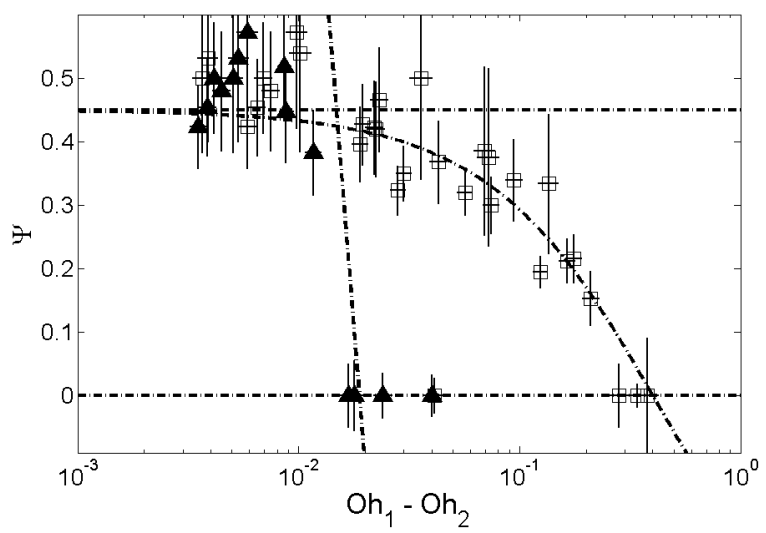

Figure 10. Semi-log plot of $\Psi$ as a function of $O h_{1}$ (triangles) and $O h_{2}$ (squares) influences on $\Psi$, when $B o<0.1$ and the other Ohnesorge number is smaller than $7.5 \cdot 10^{-3}$. Dashed lines are guides for the eyes.

\section{E. Influence of Viscosities}

Viscosity forces in both fluids, mainly efficient for small droplets, inhibit the partial coalescence $[20,21,23,28]$. The ratio $\Psi$ also monotonically decreases with both increasing Ohnesorge numbers (Fig. 10). Critical Ohnesorge numbers $O h_{1 \mathrm{c}} \sim 0.02$ and $O h_{2 \mathrm{c}} \sim 0.3$ may be defined as the Ohnesorge numbers beyond which coalescence becomes total $[20,21,23,28]$. Viscosity in fluid 2 leads to a smooth decreasing between the asymptotic regime $\Psi=0.45$ and the total coalescence $\Psi=0$. Surprisingly, the behaviour of $\Psi$ with increasing $O h_{1}$ is totally different: the decrease of $\Psi$ is very sharp and premature. The critical Ohnesorge number is 15 times smaller in fluid 1 than in fluid 2.

Capillary waves are damped by viscosity, either inside or outside the droplet. This damping can be seen through the maximum height $H_{\max }$ that is reached by the 


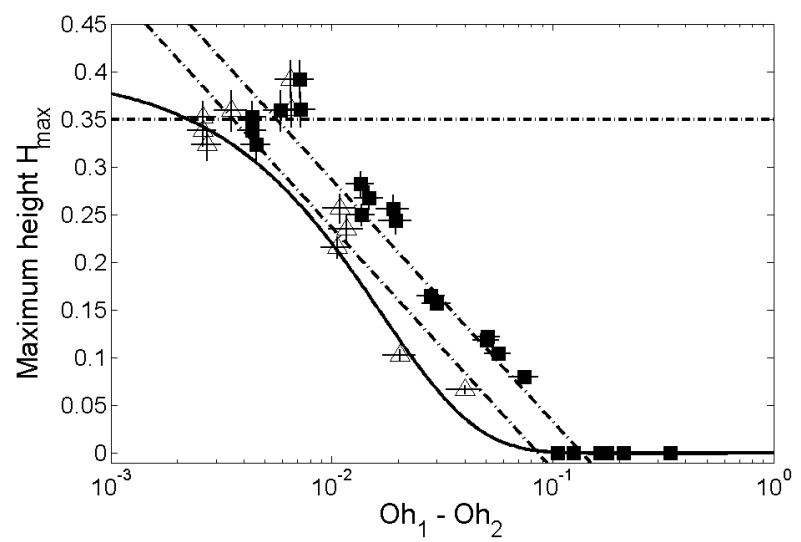

Figure 11. Maximum droplet elevation $H_{\max } v s$. $O h_{1}$ (triangles) and $O h_{2}$ (squares). Dashed lines are guides for the eyes. The solid line corresponds to the forecast of the linear waves theory.

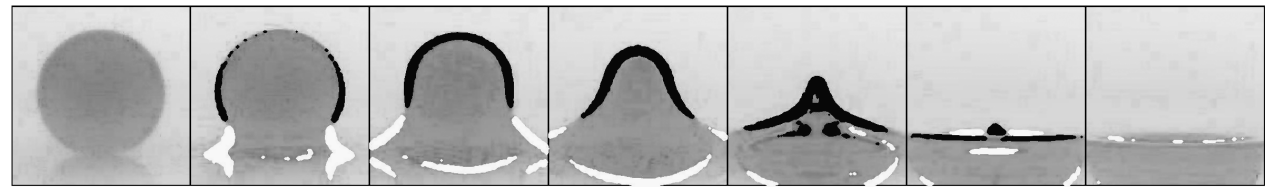

Figure 12. A total coalescence due to $\mathrm{Oh}_{2}>\mathrm{Oh}_{2 \mathrm{c}}$. Capillary waves are fully damped and no convergence is observed.

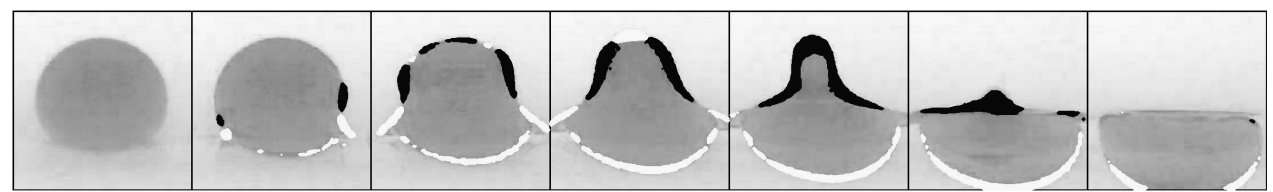

Figure 13. A total coalescence due to $O h_{1}>O h_{1 c}$. Capillary waves are still efficient, convergence is observed, but the vertical collapse is not delayed.

interface during the convergence (Fig. 11). Since the convergence effect is reduced by viscous damping, Blanchette [23] supposed that the capillary wave damping was responsible for the partial to total transition when Ohnesorge numbers are increased (Fig. 12). But it can be seen in Fig. 11 that both viscosities damp the waves in the same way: viscosity in fluid 1 is only 1.5 times more efficient than in fluid 2 . This cannot explain the asymmetrical role played by $O h_{1}$ and $O h_{2}$.

Although the delay in vertical collapse due to the convergence of capillary waves is important to get a partial coalescence, it cannot be the only determinant factor. Indeed, as seen in the snapshot of Fig. 13, a coalescence with $O h_{1}>O h_{1 \mathrm{c}}$ is total, despite the presence of capillary waves. On the other hand, in Fig. 14, a coalescence with $O h_{2}<O h_{2 c}$ is partial although the capillary waves are fully damped.

Therefore, capillary wave damping cannot be the only viscous mechanism responsible for the partial to total transition. Gilet et al. [28] recently proposed another 


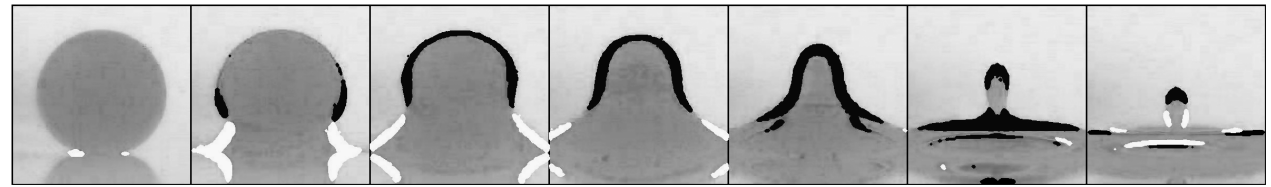

Figure 14. A partial coalescence with $O h_{2} \leqslant O h_{2 c}$, although capillary waves are fully damped and no convergence is observed.

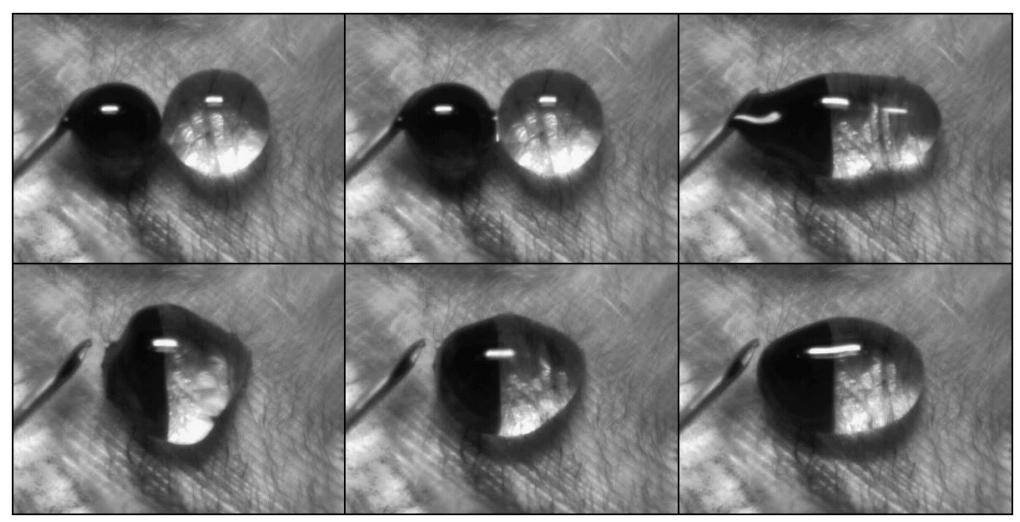

5

mechanism that is based on the repartition of the released interfacial energy between both fluids according to their ability to diffuse their kinetic energy. This hypothetical mechanism has still to be checked by PIV experiments.

\section{F. Mixing Droplets Together}

When two droplets made from miscible fluids collide with each other, they may coalesce to form a bigger droplet. As in the case of a droplet onto a planar interface, the process is not spontaneous since the film of surrounding fluid between both droplets has to be drained out. The binary coalescence of droplets together is particularly interesting on a microfluidic point of view. If reactive substances are put in each droplet, their coalescence triggers a chemical reaction in the resulting droplet [39]. The reaction kinetics is determined by the ability of both fluids to mix together. As seen in Fig. 15, this ability is really weak when droplets have a similar size: no mixing occurs, excepted by molecular diffusion. The reaction only occurs at the pseudo-interface between both fluids. The only way to enhance the reaction is to shake the droplet to create mixing flows inside.

The situation becomes interesting when two droplets with a different size coalesce. Indeed, due to a difference of Laplace overpressure, the smallest droplet fully empties into the biggest instead of simply sticking next to it. During the emptying, vorticity is generated near the moving interface [30,36-38]. As in the coalescence of a single droplet onto a planar interface, this vortex sheet may be able to separate 


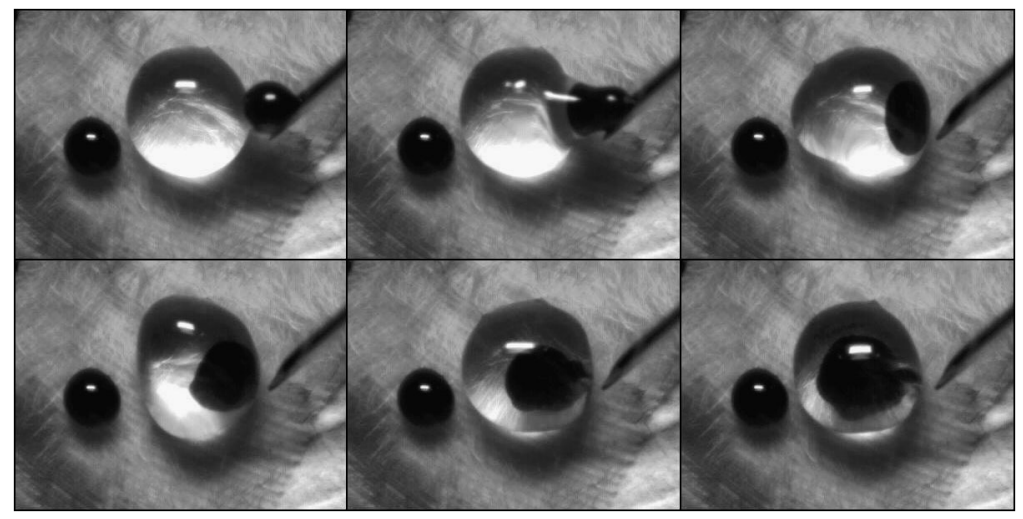

Figure 16. When two droplets with different sizes coalesce with each other, the smallest one empties into the biggest one, and creates a powerful vortex ring that efficiently mixes both fluids.

from the interface and to come deeply into the resulting droplet. The vortex ring completely mixes both liquids, as seen in Fig. 16. Conditions for this separation to occur are detailed in [38] for the planar case, but are not described yet for the binary coalescence. It seems that at least about $25 \%$ of relative difference in radius is needed for mixing.

\section{G. Control of the Partial Coalescence with Bouncing}

As seen in Section C, droplets are able to bounce indefinitely on a vertically vibrated bath. The air film between the droplet and the bath prevents the droplet from coalescence: it is constantly renewed by the vibration. The vertically vibrated bath is an excellent platform to set up microfluidic experiments. For example, it was recently shown [40] that partial coalescence can take place on this kind of bath under certain conditions. More precisely, a droplet partially coalesces when its viscosity is sufficiently weak (typically less than $5 \mathrm{cSt}$ for a millimetric oil droplet). The Faraday acceleration threshold $\Gamma_{\mathrm{F}}$ is about $1 \mathrm{~g}$ for a forcing frequency of $100 \mathrm{~Hz}$ and a viscosity of $3 \mathrm{cSt}$ : much less than the threshold for bouncing $\Gamma_{\mathrm{C}}$. The presence of Faraday waves makes the bouncing chaotic and metastable; and this presence cannot be avoided for low-viscosity droplets on a low-viscosity bath. To observe partial coalescence, it is thus more convenient to use a high-viscosity liquid for the bath, in order to significantly increase the Faraday threshold. Typically, a droplet made of $0.65 \mathrm{cSt}$ silicone oil coalesces partially on a $1000 \mathrm{cSt}$ bath vibrating at $100 \mathrm{~Hz}$. In such a system, the coalescing droplet forms a pool at the bath surface. The liquid of this pool cannot immediately mix with the surrounding oil. Consequently, the daughter droplet of the partial coalescence has unavoidably to make its firsts bounces on the pool formed by its own mother droplet. However, this does not disturb the bouncing too much.

As observed by Couder [13], the bouncing threshold $\Gamma_{\mathrm{C}}$ increases with the droplet radius. For a given acceleration, one expects that small droplets can bounce 


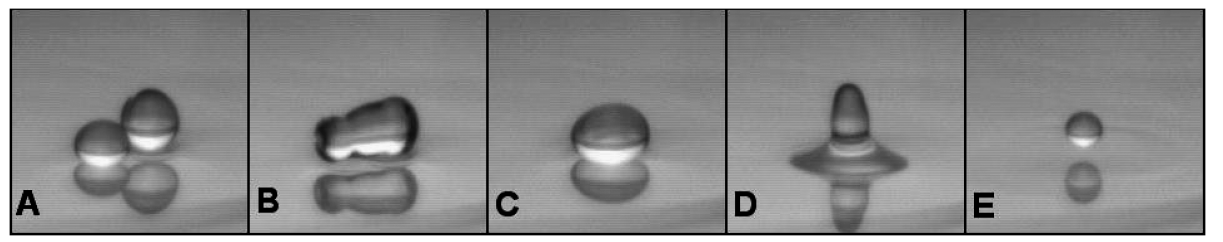

Figure 17. (A) Two droplets are bouncing close to each other on a vertically vibrated bath. (B) Suddenly, they coalesce together. (C) The resulting droplet is too large to bounce indefinitely, so (D) it partially coalesces. (E) The daughter droplet is sufficiently small to bounce.

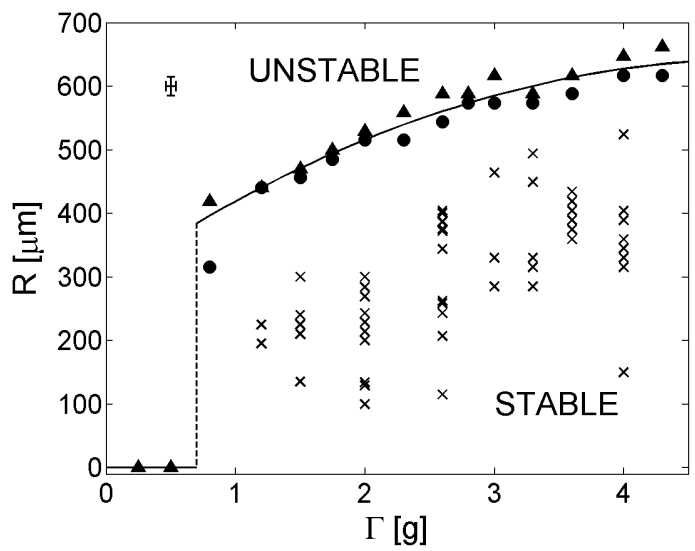

Figure 18. Critical size for bouncing as a function of the reduced vertical acceleration of the bath $\Gamma$. Droplets with radii below this size are able to bounce (stable region) while others cannot and have to empty by the way of a partial coalescence.

while large ones cannot. The partial coalescence is then stopped when the daughter droplets are sufficiently small to bounce. The whole system behaves like a low-pass filter that decreases the size of droplets until they reach a suitable size for bouncing. In Fig. 17, (A) two droplets are bouncing side by side. Suddenly, (B) they coalesce together, but $(\mathrm{C})$ the resulting droplet is too large to bounce indefinitely: (D) it coalesces partially. (E) The daughter droplet is sufficiently small to bounce periodically.

The critical size that enables bouncing has been investigated in [40]. It is shown in Fig. 18 as a function of the reduced acceleration of the bath $\Gamma$. Since the curve saturates for large $\Gamma$, it seems impossible to stabilize larger droplets by increasing the acceleration further.

\section{H. Conclusion}

In this work, several methods have been described to manipulate a droplet without touching it. The basic idea is to lay the droplet on a vibrating bath of the same liquid or of a high viscosity liquid. Conditions for bouncing have been described and low frequency vibrations provide longer lifetime. The partial coalescence is 
a nice way to reduce the size of a droplet without using complex systems. The viscosity limitations have been exposed and discussed. In order to mix droplets, we have shown that the size difference is considered as the most relevant parameter as soon as no additional means are requested. Finally, by combining the bouncing effect and the partial coalescence, the size of the droplets may be automatically selected provided it satisfies the limitations imposed by both bouncing and partial coalescence.

\section{Acknowledgements}

TG and SD thank FNRS/FRIA for financial support. Part of this work has been supported by Colgate-Palmolive (G. Broze). H. Caps is acknowledged for fruitful discussions. J.P. Lecomte (Dow Corning, Seneffe) is also thanked for the support and for providing silicone oils. This work has been supported by the COST action P21 "Physics of droplets".

\section{J. References}

1. H.A. Stone, A.D. Stroock and A. Ajdari, Annu. Rev. Fluid Mech., 36 (2004) 381.

2. N. Vandewalle, D. Terwagne, K. Mulleners, T. Gilet and S. Dorbolo, Phys. Fluids, 18 (2006) 091106.

3. W. Gonzalez-Vinas and J. Salan, Eur. Lett., 41 (1998) 159.

4. Y. Couder and E. Fort, Phys. Rev. Lett., 97 (2006) 154101.

5. A. Eddi, E. Fort, F. Moisy and Y. Couder, Phys. Rev. Lett., 102 (2009) 240401.

6. O. Reynolds, Philos. Trans. R. Soc. London Ser. A, 177 (1886) 157.

7. J. Israelachvili, Intermolecular and Surface Forces, Amsterdam, Academic Press, 2003.

8. S. Dorbolo, E. Reyssat, N. Vandewalle and D. Quéré, Europhys. Lett., 69 (2005) 966.

9. A.F. Jones and S.D.R. Wilson, J. Fluid Mech., 87 (1978) 263.

10. I.B. Ivanov and T.T. Traykov, Int. J. Multiphase Flow, 2 (1976) 397.

11. G.P. Neitzel and P. Dell'Aversana, Annu. Rev. Fluid Mech., 34 (2002) 267.

12. J.S. Eow and M. Ghadiri, Colloids Surf. A, 215 (2003) 101.

13. Y. Couder, E. Fort, A. Boudaoud and C.H. Gautier, Phys. Rev. Lett., 94 (2005) 177801.

14. T. Gilet, D. Terwagne, N. Vandewalle and S. Dorbolo, Phys. Rev. Lett., 100 (2008) 167802 (4 pages).

15. S. Dorbolo, D. Terwagne, N. Vandewalle and T. Gilet, N. J. of Phys., 10 (2008) 113021 (9 pages).

16. D. Terwagne, T. Gilet, N. Vandewalle and S. Dorbolo, Phys. of Fluids, 21 (2009) 054103 (5 pages).

17. D. Terwagne, N. Vandewalle and S. Dorbolo, Phys. Rev. E, 76 (2007) 056311 (6 pages).

18. L.D. Mahajan, Phil. Mag., 10 (1930) 383.

19. G.E. Charles and S.G. Mason, J. Colloid Sci., 15 (1960) 105.

20. Y. Leblanc, Etude des mécanismes de la coalescence partielle d'une goutte à une interface liquideliquide (Université Paris VII, 1993).

21. S.T. Thoroddsen and K. Takehara, Phys. Fluids, 12 (2000) 1265.

22. P. Pikhitsa and A. Tsargorodskaya, Colloids Surf. A, 167 (2000) 287.

23. F. Blanchette and T.P. Bigioni, Nat. Phys., 2 (2006) 1.

24. E.M. Honey and H.P. Kavehpour, Phys. Rev. E, 73 (2006) 027301.

25. H. Aryafar and H.P. Kavehpour, Phys. Fluids, 18 (2006) 072105. 
26. X. Chen, S. Mandre and J.J. Feng, Phys. Fluids, 18 (2006) 051705.

27. P. Yue, C. Zhou and J.J. Feng, Phys. Fluids, 18 (2006) 102102.

28. T. Gilet, K. Mulleners, J.P. Lecomte, N. Vandewalle and S. Dorbolo, Phys. Rev. E, 75 (2007) 036303.

29. Z. Mohamed-Kassim and E.K. Longmire, Phys. Fluids, 16 (2004) 2170.

30. B.S. Dooley, A.E. Warncke, M. Gharib and G. Tryggvason, Exp. Fluids, 22 (1997) 369.

31. S. Hartland, Trans. Inst. Chem. Eng., 45 (1967) T109.

32. J. Eggers, J.R. Lister and H.A. Stone, J. Fluid Mech., 401 (1999) 293.

33. A. Menchaca-Rocha, A. Martinez-Davalos and R. Nunez, Phys. Rev. E, 63 (2001) 046309.

34. M. Wu, T. Cubaud and C.-M. Ho, Phys. Fluids, 16 (2004) L51.

35. S.T. Thoroddsen, K. Takehara and T.G. Etoh, J. Fluid Mech., 527 (2005) 85.

36. A.V. Anilkumar, C.P. Lee and T.G. Wang, Phys. Fluids A, 3 (1991) 2587.

37. P.N. Shankar and M. Kumar, Phys. Fluids, 7 (1995) 737.

38. R.W. Cresswell and B.R. Morton, Phys. Fluids, 7 (1995) 1363.

39. H. Song, D.L. Chen and R.F. Ismagilov, Angew. Chem. Int. Ed., 45 (2006) 7336.

40. T. Gilet, N. Vandewalle and S. Dorbolo, Phys. Rev. E 76 (2007), 035302R (4 pages). 15 